\title{
Estudo do sono e função pulmonar em pacientes obesos mórbidos
}

\author{
Sleep study and pulmonary function in morbidly obese
}

\section{Isabella de Carvalho Aguiar ${ }^{[a]}$, Israel dos Santos dos Reis ${ }^{[b]}$, Wilson Rodrigues Freitas Junior ${ }^{[c]}$, Carlos Alberto Malheiros ${ }^{[\mathrm{d}]}$, Rafael Melillo Laurino Neto ${ }^{[\mathrm{e}]}$, Luis Vicente Franco de Oliveira ${ }^{[\mathrm{f}]}$}

[a] Fisioterapeuta, mestranda do Programa de Mestrado e Doutorado em Ciências da Reabilitação, Universidade Nove de Julho (Uninove), São Paulo, SP - Brasil, e-mail: isabella.carvalhoaguiar@gmail.com

[b] Fisioterapeuta, mestrando do Programa de Mestrado e Doutorado em Ciências da Reabilitação, Universidade Nove de Julho (Uninove), São Paulo, SP - Brasil, e-mail: ireissantos@uol.com.br

[c] Médico do Departamento de Cirurgia da Santa Casa de Misericórdia, São Paulo, SP - Brasil, e-mail: wrfjr@uol.com.br

[d] Médico do Departamento de Cirurgia da Santa Casa de Misericórdia, São Paulo, SP - Brasil, e-mail: camalheiros@terra.com.br

[e] Médico, coordenador do Grupo de Cirurgia Bariátrica do Conjunto Hospitalar do Mandaqui, São Paulo, SP - Brasil, e-mail: rmlaurino@uninove.br

[f] Fisioterapeuta, professor do Programa de Mestrado e Doutorado em Ciências da Reabilitação, Universidade Nove de Julho (Uninove), São Paulo, SP - Brasil, e-mail: oliveira.lvf@uninove.br

\section{Resumo}

Introdução: A obesidade acarreta uma série de alterações na fisiologia respiratória e no sono. Seu tratamento tem como objetivo a melhora da saúde e da qualidade de vida. Objetivo: Avaliar a função pulmonar e o sono em indivíduos obesos mórbidos pré-cirurgia bariátrica. Materiais e métodos: Participaram deste estudo 38 pacientes, recrutados em dois serviços de cirurgia bariátrica e encaminhados ao Laboratório de Sono da Universidade Nove de Julho, São Paulo, Brasil. Os critérios de inclusão foram: obesos mórbidos, IMC entre $40 \mathrm{~kg} / \mathrm{m}^{2}$ e $50 \mathrm{~kg} / \mathrm{m}^{2}$ e IMC entre $35 \mathrm{~kg} / \mathrm{m}^{2}$ a 39,9 kg/m² se associados a comorbidades. Resultados: A média de idade foi de $42 \pm 10$, o índice de massa corpórea médio foi de 50,09 \pm 7,64. A média da circunferência abdominal foi de 132,48 $\pm 11,07$ e 134,31 $\pm 16,26$ e de pescoço foi 42,34 $\pm 2,08$ e 44,48 $\pm 3,67$, respectivamente para mulheres e homens. As pres-

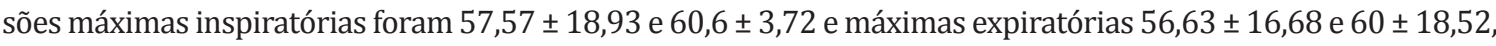
para mulheres e homens respectivamente. 0 sono do movimento rápido dos olhos apresentou-se com média de $16,93 \pm 13,61$ e a saturação mínima da oxi-hemoglobina foi de 79,33 $\pm 10,26$ durante o sono. Em 44,74\% dos casos examinados, foram observadas alterações na Escala de Sonolência de Epworth (ESE); e em 76,3\% ficou confirmada a presença de síndrome da apneia obstrutiva do sono (SAOS). Conclusão: Foram observadas alterações 
nas pressões máximas ventilatórias, na estrutura do sono associadas a considerável dessaturação noturna da oxi-hemoglobina, o que evidencia alta prevalência de SAOS nos pacientes obesos mórbidos.

Palavras-chave: Obesidade. Polissonografia. Força muscular.

\section{Abstract}

Introduction: Obesity causes a series respiratory physiology and sleep changes. Its treatment aims to improve health and quality of life. Objective: To evaluate pulmonary function and sleep in morbidly obeses pre-bariatric surgery. Materials and methods: The study had 38 patients recruited at two bariatric surgery services and referred to the Laboratório do Sono da Universidade Nove de Julho (Uninove), Sao Paulo, Brazil. The adopted criteria were: BMI between $40 \mathrm{~kg} / \mathrm{m}^{2}$ and $50 \mathrm{~kg} / \mathrm{m}^{2}$ and BMI between $35 \mathrm{~kg} / \mathrm{m}^{2}$ to 39.9 $\mathrm{kg} / \mathrm{m}^{2}$ associated comorbidities. Results: Mean age was $42 \pm 10$, the mean body mass index was $50.09 \pm$ 7.64. The average waist circumference was $132.48 \pm 11.07$ and $134.31 \pm 16.26$; the circumference neck was $42.34 \pm 2.08$ and $44.48 \pm 3.67$, respectively for women and men. The maximum inspiratory pressures were $57.57 \pm 18.93$ and $60.6 \pm 3.72$ and $56.63 \pm 16.69$ maximal expiratory and $60 \pm 18.52$ for women and men, respectively. The rapid eye movement sleep presented a mean of $16.93 \pm 13.61$ and minimum oxy-hemoglobin saturation of $79.33 \pm 10.26$ during sleep. In $44.74 \%$ of the cases studied changes were observed in the Epworth Sleepiness Scale; and in $76.30 \%$ the presence of the syndrome of obstructive sleep apnea (OSA) was confirmed. Conclusion: We observed changes in maximum pressure ventilation in sleep structure associated with significant nocturnal desaturation of oxy-hemoglobin showing a high prevalence of OSA in morbidly obese patients.

Keywords: Obesity. Polysomnographic. Muscle strength.

\section{Introdução}

À medida que se consegue erradicar a miséria entre as camadas mais pobres da população, a obesidade desponta como um problema mais frequente do que a desnutrição. Esse é um dos mais graves problemas de saúde pública da atualidade. Sua prevalência vem crescendo acentuadamente nas últimas décadas, até mesmo nos países em desenvolvimento, se tornando uma epidemia global $(1,2)$.

A obesidade é caracterizada pelo acúmulo excessivo de gordura corporal de forma a comprometer a saúde. É uma doença multifatorial que se desenvolve pela interação de fatores genéticos, ambientais, sociais, econômicos, culturais, psicológicos, entre outros (3). Em sujeitos adultos de ambos os gêneros, o sobrepeso é caracterizado por um índice de massa corpórea (IMC) de $25 \mathrm{~kg} / \mathrm{m}^{2}$ a $29,9 \mathrm{~kg} / \mathrm{m}^{2}$ e a obesidade, definida a partir de $30 \mathrm{~kg} / \mathrm{m}^{2}$ (4).

Quando o excesso de peso atinge valores elevados, com IMC $\geq 40 \mathrm{~kg} / \mathrm{m}^{2}$, a obesidade passa a ser considerada uma grave disfunção, em função da associação a doenças causadas ou agravadas por ela, correspondendo à obesidade grau III, também denominada obesidade mórbida (4). Dentre as comorbidades mais frequentes, encontram-se hipertensão arterial sistêmica $(5,6)$, diabetes mellitus tipo II (7), síndrome da apneia obstrutiva do sono (SAOS) (8), artropatias degenerativas (9), dislipidemia e coronariopatias $(10,11)$, disfunções respiratórias (12) e desajustes psicossociais (13).

A obesidade causa uma série de alterações da mecânica ventilatória, como a diminuição da capacidade funcional respiratória (CRF), do volume de reserva expiratório (VRE), da capacidade vital (CV) e da capacidade pulmonar total (CPT). 0 trabalho respiratório aumenta como consequência de uma redução da complacência torácica, aumento da resistência das vias aéreas, posição anormal do diafragma e obstrução ao fluxo aéreo em vias aéreas altas (14).

0 fator de risco mais importante para SAOS é a obesidade, observada em 60 a $70 \%$ dos pacientes, principalmente em virtude do acúmulo de gordura na porção alta do abdômen. A incidência dos distúrbios respiratórios do sono em pacientes portadores de obesidade mórbida é de 12 a 30 vezes maior do que na população geral (15). 
O padrão ouro para o diagnóstico da SAOS é a polissonografia basal noturna (PSG), caracterizada pelo registro simultâneo de parâmetros fisiológicos durante uma noite de sono em ambiente laboratorial. Por esse exame, é possível analisar a arquitetura do sono, o padrão respiratório, a saturação da oxi-hemoglobina, a função cardiovascular e os movimentos corporais (16).

0 tratamento da obesidade tem como objetivo a melhora da saúde e da qualidade de vida, mediante suficiente redução do peso corporal para eliminar ou reduzir as comorbidades e promover o bem-estar psicológico. Isso constitui um atributo da cirurgia bariátrica, visto que a obesidade mórbida permanece largamente refratária à terapêutica dietética e medicamentosa (17).

0 presente estudo objetiva avaliar a estrutura do sono e a função pulmonar em indivíduos portadores de obesidade mórbida com indicação de cirurgia bariátrica.

\section{Materiais e métodos}

Esta pesquisa é um estudo transversal, registrado pelo World Health Organization Universal Trial Number (UTN) U1111-1121-8873, pelo Registro Brasileiro de Ensaios Clínicos (RBR-9k9hhv), e aprovado pelo Comitê de Ética e Pesquisa da Universidade Nove de Julho (Uninove) sob o protocolo n. 220506/2009.

Os pacientes portadores de obesidade mórbida (IMC entre $40 \mathrm{~kg} / \mathrm{m}^{2}$ e $50 \mathrm{Kg} / \mathrm{m}^{2}$ ) e com IMC entre 35 $\mathrm{kg} / \mathrm{m}^{2}$ a $39,9 \mathrm{~kg} / \mathrm{m}^{2}$ associado a comorbidades foram recrutados do Serviço de Cirurgia Gástrica da Santa Casa de Misericórdia de São Paulo e do Grupo de Cirurgia Bariátrica do Conjunto Hospitalar do Mandaqui, em São Paulo, e encaminhados ao Laboratório de Sono da Universidade Nove de Julho (Uninove).

Participaram do estudo 38 pacientes, de ambos os gêneros - 33 mulheres e 5 homens. Os critérios de inclusão foram: indivíduos portadores de obesidade mórbida (IMC entre $40 \mathrm{~kg} / \mathrm{m}^{2}$ e $50 \mathrm{Kg} / \mathrm{m}^{2}$ ), com IMC entre $35 \mathrm{~kg} / \mathrm{m}^{2}$ e $39,9 \mathrm{~kg} / \mathrm{m}^{2}$ associado a comorbidades e que concordaram em participar do estudo, assinando o Termo de Consentimento Livre e Esclarecido (TCLE).

Os critérios de exclusão adotados foram: uso abusivo de álcool e uso de medicamentos que interferem no eletroencefalograma, como hipnóticos e estimulantes do sistema nervoso central.
A avaliação dos sujeitos foi realizada no Laboratório do Sono da Universidade Nove de Julho (Uninove). Essa avaliação consistiu em coleta de dados pessoais do paciente, seguida de verificação dos seguintes itens: frequência cardíaca, frequência respiratória, pressão arterial sistêmica periférica, peso e altura, IMC, cirtometria do pescoço e abdômen, espirometria, manovacuometria, polissonografia basal noturna. Nessa oportunidade, procedeu-se também a aplicação do Questionário Clínico de Berlin e da Escala de Sonolência de Epworth, de acordo com protocolo de pesquisa previamente publicado (18).

As referências anatômicas das mensurações de circunferência de cintura e pescoço foram padronizadas. Na cintura pélvica, a circunferência foi verificada entre a margem inferior da última costela e a crista ilíaca; a circunferência do pescoço foi medida horizontalmente no nível da cartilagem cricoide (19).

Os testes de função pulmonar foram realizados com o paciente sentado em posição cômoda, com o uso do espirômetro KoKo PFT System Versão 4.11 (nSpire Health, Inc; Louisville, CO, USA); os testes seguiram as diretrizes nacionais para a realização de provas de função pulmonar da Sociedade Brasileira de Pneumologia e Tisiologia (20). Para a mensuração das pressões máximas geradas pelos músculos ventilatórios, foi utilizado o manovacuômetro aneroide da marca Ger-Ar (Ger-Ar Com. Produtos Médicos Ltda., São Paulo, Brasil). Os valores previstos utilizados foram os de Neder et al. (21).

Para os estudos do sono, foi utilizada a monitorização padrão com o sistema ambulatorial de análise do sono modelo Embla (A10 versão 3.1.2 Flaga, Hs. Medical Devices, Reykjavík, Islândia), composto de 16 canais, eletroencefalograma, eletro-oculograma, eletromiograma, fluxo aéreo nasal e oral, esforço ventilatório torácico e abdominal, oximetria digital de pulso e eletrocardiograma. Todos os sinais foram continuamente gravados durante todo o período de sono de cada paciente e acompanhados por um experiente técnico em polissonografia (22).

A SAOS é definida como a cessação completa do fluxo aéreo por dez segundos ou mais associada a uma dessaturação da oxi-hemoglobina de pelo menos 3\%. A hipopneia, por sua vez, é caracterizada por significante redução do fluxo aéreo ( $\geq 50 \%)$ por pelo menos dez segundos associada a uma dessaturação da oxi-hemoglobina de 3\% ou mais. 0 índice de apneia/ hipopneia é obtido pelo quociente da operação total de eventos respiratórios/hora de sono. As polissonografias 
foram estagiadas manualmente, em épocas de 30 segundos; os laudos técnicos foram confeccionados por médico especialista em Medicina do Sono (22).

Foi utilizada a estatística descritiva para interpretação dos dados, expressos em média e desvio padrão, para os dados paramétricos. Para os valores não paramétricos foi utilizada mediana e suas variações máximas e mínimas. As correlações entre as variáveis foram feitas pelo teste de Pearson. 0 nível de significância estatística foi definido em $5 \%$ para todos os testes $(\mathrm{p} \leq 0,05)$. 0 pacote estatístico utilizado foi o SPSS 19.0 (Chicago, IL, USA).

\section{Resultados}

Inicialmente foram convidados a participar deste estudo 49 pacientes portadores de obesidade mórbida em lista de espera para cirurgia bariátrica de dois hospitais. No decorrer do estudo, 11 pacientes não realizaram as avaliações por não atenderem aos critérios de inclusão. Por esse motivo, a amostra final do estudo foi composta por 38 pacientes. As características antropométricas e a média da idade estão descritas na Tabela 1 , a seguir.

As pressões ventilatórias máximas (PImáx e PEmáx) comparadas com os valores previstos pelas equações de Neder et al. (21) e os valores espirométricos em porcentagem do predito estão apresentadas na Tabela 2.

Observa-se na amostra avaliada um índice médio de eventos respiratórios obstrutivos (IAH) de $24,97 \pm 29,23$, caracterizado como SAOS moderada.

Tabela $\mathbf{l}$ - Valores antropométricos

\begin{tabular}{|c|c|c|c|}
\hline Variáveis & & & $(n=38)$ \\
\hline Idade (anos) & & & $42 \pm 10$ \\
\hline Altura (cm) & & & $1,60 \pm 0,09$ \\
\hline Peso (Kg) & & & $124,36 \pm 24,42$ \\
\hline IMC (Kg/m2) & & & $50,09 \pm 7,64$ \\
\hline \multirow{4}{*}{ Circunferência (cm) } & \multirow{2}{*}{ Abdominal } & Feminino & $132,48 \pm 11,07$ \\
\hline & & Masculino & $134,31 \pm 16,26$ \\
\hline & \multirow{2}{*}{ Pescoço } & Feminino & $42,32 \pm 2,08$ \\
\hline & & Masculino & $4,48 \pm 3,67$ \\
\hline
\end{tabular}

Legenda: IMC = Índice de massa corpórea.

Fonte: Dados da pesquisa.
Entretanto, vale salientar que observou-se também um considerável desvio padrão de 29,23, que indica um grande percentual de pacientes com apneia grave.

A Tabela 3, a seguir, apresenta as variáveis polissonográficas observadas.

A Tabela 4 mostra os resultados da aplicação da Escala de Sonolência de Epworth, sendo observado um valor médio de 6 , com variações de 0 a 24 .

A Figura 1 mostra a correlação de índice de apneia/hipopneia e índice de massa corpórea, índice de apneia/hipopneia e idade, índice de apneia/hipopneia e circunferência do pescoço que apresentaram respectivamente os valores de $\mathrm{p}=0,15 / \mathrm{R}=0,23, \mathrm{p}=$ $0,009 / R=0,42$ e $p=0,001 / R=0,53$. A análise da figura evidencia que, apesar de os valores de p não apresentarem significância ( $p>0,5$ ), houve uma correlação positiva nas situações referidas.

Tabela 2 - Espirometria e pressões máximas ventilatórias

\begin{tabular}{|c|c|c|c|}
\hline$(n=38)$ & & Obtido & Previsto \\
\hline CVF(\%) & & $100,13 \pm 21,18$ & \\
\hline $\operatorname{VEF}_{1}(\%)$ & & $97,47 \pm 21,00$ & \\
\hline $\begin{array}{l}\mathrm{VEF}_{1} / \\
\text { CVF(\%) }\end{array}$ & & $80,00 \pm 07,00$ & \\
\hline \multirow{2}{*}{ PImax } & Feminino & $57,57 \pm 18,93$ & $86,53 \pm 8,76$ \\
\hline & Masculino & $60,6 \pm 3,72$ & $116,78 \pm 14,02$ \\
\hline \multirow{2}{*}{ PEmax } & Feminino & $56,63 \pm 16,69$ & $85,88 \pm 10,09$ \\
\hline & Masculino & $60 \pm 18,52$ & $126,30 \pm 14,19$ \\
\hline
\end{tabular}

Legenda: CVF = Capacidade Vital Forçada; VEF1 = Volume expiratório forçado no primeiro segundo; VEF1/CVF = Índice de Tifenneau; PImáx = Pressão inspiratória máxima; PEmáx = Pressão expiratória máxima.

Fonte: Dados da pesquisa.

Tabela 3 - Variáveis polissonográficas

(Continua)

\begin{tabular}{lc} 
Variáveis & $(\mathbf{n}=\mathbf{3 8})$ \\
\hline (\%) Efic. Sono & $73,29 \pm 16,87$ \\
NREM-1(\%) & $9,94 \pm 9,64$ \\
NREM-2(\%) & $44,28 \pm 18,25$ \\
NREM-3(\%) & $17,48 \pm 18,37$
\end{tabular}


Tabela 3 - Variáveis polissonográficas

\begin{tabular}{lc}
\hline Variáveis & $(\mathbf{n}=\mathbf{3 8})$ \\
\hline REM (\%) & $16,93 \pm 13,61$ \\
$\mathrm{SpO}_{2}$ média & $92,53 \pm 4,02$ \\
$\mathrm{SpO}_{2}$ mínima & $81,73 \pm 9,09$ \\
Índice Despertar & $7,36 \pm 7,33$ \\
\% IAH normal $(<5)$ & 23,68 \\
\% IAH leve (5-15) & 26,31 \\
\% IAH moderado (16-30) & 23,68 \\
\% IAH grave $(>30)$ & 26,31 \\
\hline
\end{tabular}

Legenda: NREM1 = Estágio 1 do sono sem movimentos rápidos dos olhos; NREM 2 = Estágio 2 do sono sem movimentos rápidos dos olhos; NREM 3 = Estágio 3 do sono sem movimentos rápidos dos olhos; REM = Sono com movimentos rápidos dos olhos; Sp02 = Saturação periférica de oxigênio; SAOS = Síndrome da apneia obstrutiva do sono; IAH = Índice de apneia e hipopneia por hora.

Fonte: Dados da pesquisa.

Tabela 4 - Escala de sonolência de Epworth

\begin{tabular}{lcc}
\hline Escala de sonolência de Epworth & $(\mathbf{n}=\mathbf{3 8})$ & \% \\
\hline Normal & 21 & 55,26 \\
Leve & 9 & 23,68 \\
Moderada & 4 & 10,52 \\
Grave & 4 & 10,52 \\
\hline
\end{tabular}

Fonte: Dados da pesquisa.

\section{Discussão}

A obesidade atualmente é um dos problemas mais graves de saúde pública, sua prevalência vem crescendo acentuadamente nas últimas décadas. 0 presente estudo se propôs a avaliar a função pulmonar e a qualidade do sono de pacientes portadores de obesidade mórbida.

Em relação às variáveis antropométricas estudadas, observamos um valor da circunferência abdominal de 132,48 $\pm 11,07$ no grupo feminino e de 134,31 $\pm 16,26$ no grupo masculino; esses valores excedem
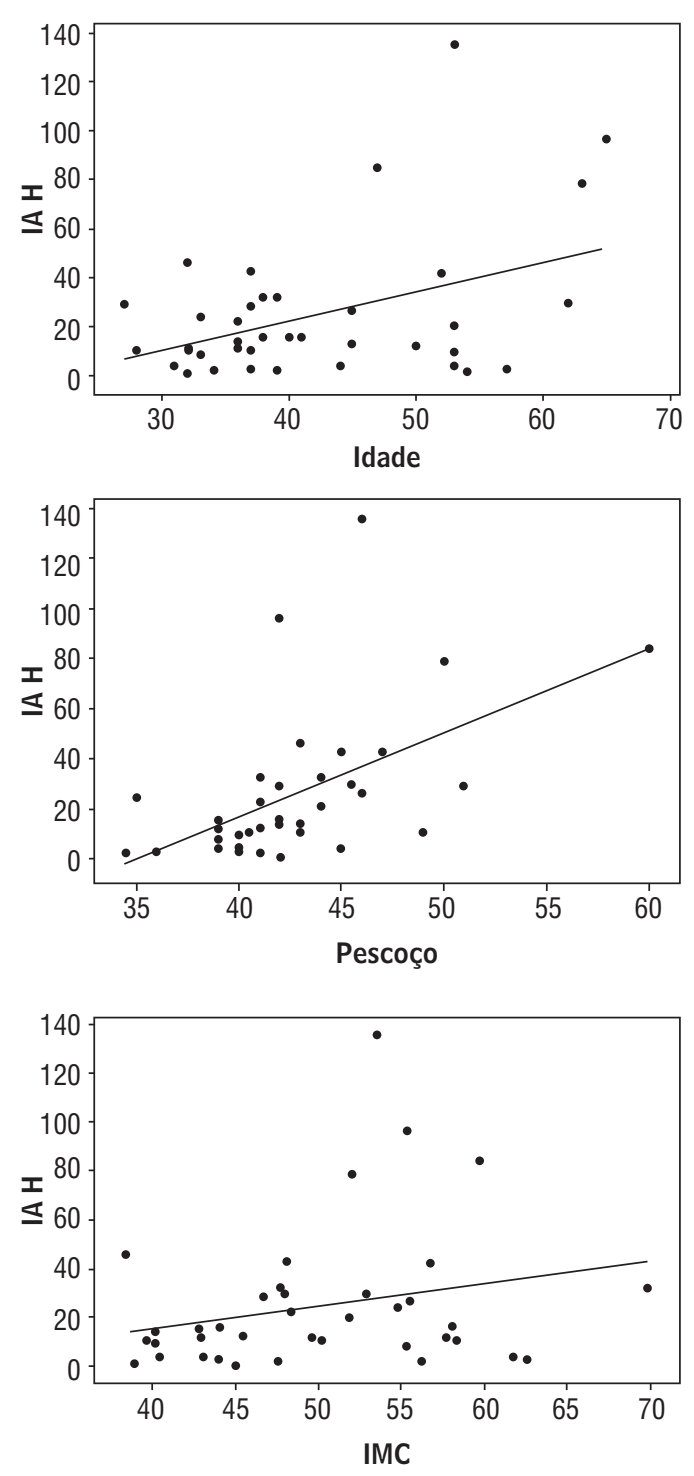

Figura 1 - Coeficiente de correlação de índice de IAH/IMC, IAH/idade e IAH/circunferência do pescoço

Fonte: Dados da pesquisa.

os valores de normalidade previstos por Ashweel et al. (23) - feminino, $\leq 80$; masculino, $\leq 94$.

Quando observados os valores referentes à circunferência de pescoço para os dois gêneros, observou-se um valor acima do ponto de corte (mulheres, $\leq 41$; homens, $\leq 43$ para homens) (23), de 42,32 $\pm 2,08$ e 44,48 $\pm 3,67$ para mulheres e homens respectivamente. A circunferência de pescoço é considerada um fator de risco para SAOS devido ao acúmulo de gordura na região do pescoço; o sexo masculino é mais afetado pelas diferenças anatômicas das vias aéreas superiores 
por seu perfil hormonal e pela distribuição adiposa do tipo central (tronco e pescoço) (24).

A avaliação da força muscular nos obesos tem valor importante, uma vez que o comprometimento desta musculatura pode implicar em complicações cardiorrespiratórias (25).

Pesquisas têm revelado que a obesidade causa distúrbios respiratórios; o sintoma de dispneia, por exemplo, se correlaciona com o grau de obesidade $(14,26)$. Um estudo, avaliando a capacidade funcional, indica que não só o peso interfere na capacidade funcional, mas também a idade, o sexo e a estatura; esta última variável foi a que melhor apresentou alteração na função pulmonar (27).

De acordo com o estudo de Soares et al. (28), com a submissão aos testes espirométricos, verificou-se que os pacientes obesos não apresentaram características obstrutivas ou restritivas avaliados pela CVF e VEF1, uma vez que os volumes e capacidades estão adequados quando comparados aos de indivíduos normais. Esse resultado se contrapõe aos obtidos pelo estudo de Ogunnaike et al. (29).

No estudo de Magnani et al. (30), do qual participaram somente obesos com idade entre 20 a 64 anos, confirmou-se que a obesidade não interfere na pressão gerada pelos músculos ventilatórios, pois os valores não apresentaram significância quando comparados aos valores de referência de normalidade indicados por Neder et al. (21).

Diferentemente dos resultados obtidos por Cardoso (31) e Magnani et al. (30), os achados do presente estudo revelaram que a força muscular ventilatória em pacientes obesos foi marcadamente reduzida quando comparada aos valores preditos descritos por Neder et al. (21).

Queiroz (32) observou resultados semelhantes aos nossos ao avaliar pacientes obesos e não obesos ( $\mathrm{n}=100)$ pareados por sexo. As pressões máximas inspiratórias e expiratórias geradas pelos músculos ventilatórios dos pacientes, independente do sexo, apresentaram valores absolutos menores quando comparados a indivíduos não obesos.

Considerando a classificação AASSM Task force (American Academy of Sleep Medicine) (22), encontramos em nossos pacientes um IAH de 76,30\%, confirmando os resultados do estudo de Berger et al. (15). Em relação à eficiência do sono, foram identificados valores abaixo da normalidade (> 85\%): $73,29 \pm 16,87$. Cabe salienar que Dixon et al. (33), em um estudo com obesos mórbidos com IMC entre
45 e $53 \mathrm{~kg} / \mathrm{m}^{2}$, encontraram uma porcentagem de eficiência do sono (71\%) menor que a encontrada em nosso estudo.

Avaliando os dados de saturação parcial de oxigênio durante a vigília e saturação média durante o sono, não foram identificadas alterações quanto aos valores de normalidade. Esse resultado confirma os achados de Busetto et al. (34), que também não encontraram alterações quanto à saturação da oxi-hemoglobina.

Com relação à ESE, 44,74 \% dos pacientes apresentaram um índice de leve a grave conforme os valores de referência (35); essa constatação ratifica o resultado do estudo de Dixon et al. (33), que encontraram um índice de 10,1 \pm 5,1 em pacientes obesos. Na pesquisa realizada por Sharkey et al. (35), com a participação de 269 pacientes, não foram encontradas alterações quanto à ESE $(6,3 \pm 4,8)$, mesmo realizada a estratificação de acordo com os níveis de gravidade. Isso indica que a ESE é uma escala subjetiva (35).

A anormalidade na função pulmonar e a qualidade do sono em pacientes obesos mórbidos têm sido estudadas há cerca de quatro décadas. Entretanto, ainda persistem algumas divergências sobre a associação de alterações pulmonares e de sono com o peso corporal ou mesmo com o índice de massa corpórea (36-38).

\section{Conclusão}

O sobrepeso e a obesidade causam prejuízo significativo nas pressões ventilatórias máximas inspiratórias e expiratórias. Os valores obtidos nessa população são inferiores aos previstos para a população brasileira. Uma considerável perda da função ventilatória pode comprometer o período intra e pós-cirúrgico, acarretando piora do prognóstico do paciente. Isso indica a relevância do treinamento da musculatura respiratória no pré-operatório de cirurgia bariátrica.

A SAOS foi observada em $76,30 \%$ dos pacientes, confirmando elevada presença de distúrbios respiratórios do sono em pacientes obesos mórbidos. Considerando esses achados, é possível sugerir o uso do PAP (pressão positiva na via aérea superior), com a finalidade de melhorar os marcadores inflamatórios e a qualidade de vida no pós-operatório de cirurgia bariátrica.

Permanecendo as divergências observadas, sugerimos a realização de outros estudos que proponham valores de referência específicos para a população de obesos mórbidos. 


\section{Referências}

1. World Health Organization - WHO. Obesity. and overweight. Fact Sheet No 311, 2006. [cited 30 Aug 2012.] Available from: http://www.who.int/mediacentre/ factsheets/fs311/en/ index.html.

2. Xavier MAF, Ceneviva R, Terra J Filho, Sankarankutty AK. Pulmonary function and quality of life in patients with morbid obesity six months after bariatric surgery. Acta Cir Bras. 2010;25(5):407-15. doi:10.1590/ S0102-86502010000500005.

3. Zammit C, Liddicoat H, Moonsie I, Makker H. Obesity and respiratory disease. Int J Gen Med. 2010;3:33543. PMid:21116339.

4. Clinical guidelines on the identification, evaluation, and treatment of overweight and obesity in adults. The evidence report: National Institutes of Health. Obes Res. 1998;Suppl 2:51S-209S. PMid:9813653.

5. Nieto FJ, Young TB, Lind BK. Association of sleep disordered breathing, sleep apnea and hypertension in a large community-based study: sleep heart health study. JAMA 2000;283(14):1829-36. doi:10.1001/ jama.283.14.1829.

6. Drager LF, Bortolotto LA, Krieger EM, Lorenzi-Filho G. Additive effects of obstructive sleep apnea and hypertension on early markers of carotid atherosclerosis. Hypertension. 2008;53(1):64-9. doi:10.1161/ HYPERTENSIONAHA.108.119420.

7. Kanaley JA, Goulopoulou SH, Franklin RM, Baynard T, Holmstrup ME, Carhart R Jr., et al. Plasticity of heart rate signaling and complexity with exercise training in obese individuals with and without type 2 diabetes. In J Obes (Lond). 2009; 33(10):1198-206.

8. Young T, Peppard PE, Taheri S. Excess weight and sleepdisordered breathing. J Appl Physiol. 2005;99(4):15929. doi:10.1152/japplphysiol.00587.2005.

9. Beyerlein A, Von Kries R, Ness AR, Ong KK. Genetic markers of obesity risk: strong associations with body composition in overweight compared to normal-weight children. Plos one. 2011;6(4):19057. doi:10.1371/journal.pone.0019057.

10. Alan I, Lewis MJ, Lewis KE, Stephens JW, Baxter JN. Influence of bariatric surgery on indices of cardiac autonomic control. Auton Neurosci. 2009;151(2):16873. doi:10.1016/j.autneu.2009.08.007.
11. Peker Y, Hedner J, Kraiczi H. Respiratory disturbance index: an independent predictor of mortality in coronary artery disease. Am J Respir Crit Care Med. 2000;162:81-6. PMid:10903224.

12. Lazarus R, Sparrow D, Weiss TS. Effects of obesity and fat distribution on ventilatory function: the normative aging study. Chest. 1997;111(4):891-8. doi:10.1378/ chest.111.4.891.

13. Blake C, Fabick KM, Seychell KDR, Lund TD, Lephart ED. Neuromodulation by soy dietsor equol: anti-depressive e anti-obesity-like influences, age e hormone-dependent effects. BMC neurosciences. 2011;12(28):1471-202.

14. Stibulov R. Repercussões respiratórias da obesidade. J Bras Pneumol. 2007;33(1):28-35.

15. Berger KI, Ayappa I, Chart-amontri B, Marfatia A, Sorkin IB, Rapoport DM. Obesity hypoventilation syndrome as a spectrum of respiratory disturbances during sleep. Chest. 2001;120(4):1231-8. doi:10.1378/ chest.120.4.1231.

16. Badr MS. Pathophysiology of upper airway obstruction during sleep. Clin Chest Med.1998;9(1):45-50.

17. Poirier P, Giles TD, Bray GA, Hong Y, Stern J, Pi-Sunyer FX, et al. Obesity and cardiovascular disease: pathophysiology, evaluation and effect of weight loss. Circulation. 2006;113(6):898-918. doi:10.1161/ CIRCULATIONAHA.106.171016.

18. Oliveira LV, Aguiar IC, Hirata RP, Faria NS Junior, Reis IS, Sampaio LM, et al. Sleep study, respiratory mechanics, chemosensitive response and quality of life in morbidly obese patients undergoing bariatric surgery: a prospective, randomized, controlled trial. BMC Surgery. 2011;11(28):1-8.

19. Gabrielsen AM, Lund MB, Kongerud J, Viken KE, Roislien J, Hjelmesaeth J. The relationship between anthropometric measures, blood gases and ling function in morbidly obese white subjects. Obes Surg. 2011;21(4):48591. doi:10.1007/s11695-010-0306-9.

20. Pereira CA. II Consenso Brasileiro de Espirometria. J Pneumol. 2002;28(Suppl 3):S1-S82.

21. Neder JA, Andreoni S, Lelario MC, Nery LE. Reference values for lung function II. Maximal respiratory pressure and voluntary ventilation. Braz J Med Biol Res. 1999; 32(6):729-37. doi:10.1590/ S0100-879X1999000600008. 
22. Iber C, Ancoli-Israel S, Quan SF. The AASM manual for the scoring of sleep and associated events: rules, terminology, and technical specifications for the American Academy of Sleep Medicine. Westchester: American Academy of Sleep Medicine; 2007. PMCid:2134956.

23. Ashweel M, Durrant M, Garrow JS. How a fat cell pool hypothesis could account the relationship between adipose tissue cellularity and the age of onset of obesity. Proc Nutr Soc. 1997;36(3):111A.

24. Mancini MC, Aloe F, Tavares S. Apnéia do sono em obesos. Arq Bras Endocrinol Metab. 2000;44(1):81-5. doi:10.1590/S0004-27302000000100013.

25. Isono, S. Obesity and obstructive sleep apnoea: mechanisms for increased collapsibility of the passive pharyngeal airway. Respirology. 2012;17:32-42. doi:10.1111/j.1440-1843.2011.02093.x.

26. Aguiar IC, Reis IR, Apostólico N, Pinto LA, Freitas WR Jr, Malheiros CA, et al. Capacidade pulmonar e força ventilatória em obesos mórbidos. Ter Man. 2012;10(47):71-4.

27. Kozeki LCC, Bertolini SMMG. Capacidade muscular e força muscular respiratória em crianças obesas. Rev Saúde e Pesq. 2011;4(2):169-76.

28. Soares KKD, Gomes ELFD, Beani A Junior, Oliveira LVF, Sampaio LMM, Costa D. Avaliação do desempenho físico e funcional respiratório em obesos. Fisioter Mov. 2011;24(4):697-704.

29. Ogunnaike BO, Jones SB, Jones DB, Provost D, Whitten CW. Anesthetic considerations for bariatric surgery. Anesth Analg. 2002;95(6):1793-805. doi:10.1097/00000539-200212000-00061.

30. Magnani KL, Cataneo AJM. Respiratory muscle strength in obese individuals and influence of upperbody fat distribution. São Paulo Med J. 2007; 125(4):215-9. doi:10.1590/S1516-31802007000400004.
31. Cardoso FPF. Manovacuometria e ventilometria de mulheres obesas no pré-operatório de gastroplastia redutora [dissertação]. Brasília, DF: Universidade Católica de Brasília; 2005.

32. Queiroz JCF. Correlação entre a força de pressão palmar e a força da musculatura respiratória em indivíduos obesos e não-obesos [dissertação]. Brasília, DF: Universidade Católica de Brasília; 2006.

33. Dixon JB, Schachter LM, O’Brien PE. Predicting sleep apnea and excessive day sleepiness in the several obesity. Chest. 2003;123(4):1134-41. doi:10.1378/ chest.123.4.1134.

34. Busetto TL. Obstructive sleep apnea syndrome in morbid obesity: effects of intragastric balloon. Chest. 2005;128:618-23. doi:10.1378/chest.128.2.618.

35. Sharkey KM, Orff HJ, Tossi C, Harrington D, Roye GD, Millman RP. Subjective sleepiness and daytime functioning in bariatric patients with obstructive sleep apnea. Sleep Breath. 2012;13:5-10.

36. Piper AJ. Obesity and respiratory disease - weighing in on the issue: an epilogue. Respirology. [Epub ahead of print]. 2012:1-7.

37. Franklin KA, Sahlin C, Stenlund H, Lindberg E. Sleep apnoea is a common occurrence in females. Eur Respir J. [Epub ahead of print]. 2012;16:21-7.

38. Kim SM, Cho KI, Kwon JH, Lee HG, Kim TI. Impact of obstructive sleep apnea on left atrial functional and structural remodeling beyond obesity. J Cardiol. 2012;60(6) 475:83.

Recebido: 26/09/2012

Received: 09/26/2012

Aprovado: 23/10/2012

Approved: 10/23/2012 